\title{
Viscous/inviscid Interacting Shear Flow Theory with Inferences and Their Applications to CFD
}

\author{
$Z_{\text {Gao }}{ }^{1}$ Y.Q.Shen ${ }^{1}$ G.C.Zha ${ }^{2}$
}

1. LHD, Institute of Mechanics, Chinese Academy of Sciences, 100190, Beijing, PRC

2. Dept.of Mechanical \& Aerospace Engineering, University of Miami, FL 33124, USA

Abstract: In the viscous/inviscid interacting shear flow(ISF) theory, interacting shear perturbed flow(ISPF) theory and interacting shear turbulent flow(ISTF) theory suggested by Gao, the ISF consists of viscous shear layer and neighboring outer inviscid flow, which interact each other. The motion laws, definition and governing equations of the above three flows are described in ISF's optimal coordinates, which is a fitted dividing flow surface orthogonal coordinates. The scaling laws of velocity and length of ISF's viscous layer are deduced the scaling laws imply the strength of viscous/inviscid flow interaction. The scaling laws of velocity and length of both ISPF's viscous perturbed layer and ISTF's viscous turbulent layer are also given. The equations governing ISF are the Parabolized Navier-Stokes(PNS) equations, which can be simplified further on the dividing surface. The resultant equations are defined as dividing flows surface criteria, whose two important special cases are wall-surface criteria for viscous and inviscid flows. The ISF's optimal coordinates and length scaling law are used to design the grid. The small scale structures given by the scaling laws can be used to predict local sudden changes of heat flux etc., which are very important for hypersonic flows. The wall-surface criteria are used to validate NS numerical solutions for ISF and flow near walls. The wall-surface criteria method has several advantages over the commonly used grid convergence criteria. The applications of ISF theory indicates its effectiveness and further studies and development are needed. 
Key words: fluid dynamics, computational fluid dynamic, viscous/inviscid interacting flow, scaling law, CFD's verification and validation

\section{Introduction}

The boundary layer theory opened up a new era of fluid mechanics. From the 1930s to the 1960s, analyses and computations of high Reynolds number flows were based on the boundary layer theory, i.e. to compute firstly inviscid flow over body and then to compute viscous thin boundary layer flow with both the no-slip wall condition and the solution of inviscid flow on the wall surface as boundary conditions. In CFD era, the boundary layer theory still has important meaning, just as Schlicting and Gersten pointed out in their monograph ${ }^{[1]}$ : "Numerical methods in computing flows at high Reynolds numbers only become efficient if the particular layered structure of the flow, as given by the asymptotic theory, is taken into account, as occur if a suitable grid is used for computation. Boundary-layer theory will therefore retain its fundamental place in the computation of high Reynolds number flows.”

From the 1960s to the 1990s, a new upsurge in studies and computations of parabolized Navier-Stokes(PNS) was in the making. For a steady supersonic flow over a body, PNS equations can be computed by space marching algorithm(SMA), that reduces the dimension number of computation. Especially, in the computation of high Reynolds number flows, only second-order normal derivatives of viscous terms in the NS 
equations written in a fitted body orthogonal coordinates can be computed accurately, other viscous terms in the NS equations cannot be computed, then a NS computations is actually a computation of thin-layer NS, i.e. PNS computation. Therefore, for the computations for engineering purpose PNS has always fundamental meaning, just as Anderson pointed out in his monograph ${ }^{[2]}$ "the PNS method is in very widespread use; indeed, it forms the basis of an industry-standard computer program, which is used by virtually all major aerodynamics laboratories and companies.”

However, it is not very clear what kind of basic flow is described by the PNS equations before the 1990s, there is no basic fluid theory corresponding PNS equations. The viscous/inviscid interacting shear flow(ISF) theory ${ }^{[3-5]}$ furnishes complete answer to this subject. ISF consists of viscous shear flow and its neighboring outer inviscid flow, which interact on each other. The equations governing ISF are just PNS equations. ISF theory forms basic fluid theory for PNS equations and it also makes a breakthrough the classical boundary layer theory, in which there is no interacting between the boundary layer and its outer inviscid flow. Especially, ISF theory and its inferences also have some important applications to CFD, such as application of the ISF's optimal coordinates to grid design, application of length scaling laws of ISF's viscous layer to grid design, using small scale structure given by the scaling-laws to 
compute sudden changes of several physical quantities, that are called “unknown-unknown” for hypersonic flow by Bertin and Cumming ${ }^{[6]}$, applications of wall-surface-criteria for laminar flow, perturbed flow and turbulent flow to verify creditabilities of NS and RANS numerical solutions and to optimize turbulent models etc. Therefore, PNS equations and ISF theory are very useful for the computations of high Reynolds number flows.

In a word, the comment of Schlichting and Gersten ${ }^{[1]}$ on the place of boundary-layer theory in CFD is similar suitable to ISF theory, that is, the computation of high Reynolds number flows only become efficient if the thin layer and small scales structures in normal, streamwise even crosswise directions as given by the scaling-laws of ISF theory can be resolved by a suitable grids with locally refined in locations occurring small scale structures, only by which the sudden change phenomena of some physical quantities can be computed accurately. In the rapid development of the computation of high Reynolds number flows, we should pay great attention to integrating computation with fluid theories.

\section{Viscous/inviscid interacting shear flow(ISF) theory}

1.1 ISF theory for laminar flow ${ }^{[3-5]}$ : Viscous/inviscid interacting shear flow (ISF) consists of viscous shear layer and neighboring outer inviscid flow, which interact on each other. The motion-law of ISF's viscous layer is convection-diffusion competitive in its normal direction, while it is 
convection-dominate in its streamwise direction. The mathematical definition of this motion-law is for a two-dimensional incompressible ISF

$$
u \frac{\partial f}{\partial x} ? \quad \lambda \frac{\partial^{2} f}{\partial x^{2}}, \quad v \frac{\partial f}{\partial y} \cong \lambda \frac{\partial^{2} f}{\partial y^{2}}
$$

where the $x$ and $y$ are coordinate variables of a fitted dividing-flow-surface orthogonal coordinates, that is called ISF's optimal coordinates, otherwise the definition formula(1) does not hold. There is always a dividing-flow-surface in ISF's viscous layer ${ }^{[1]}$, especially, the wall surface is a dividing-flow-surface of viscous flow close neighbor wall surface. $u$ and $v$ are velocity components in the $x-$ and $y$-directions, respectively. $f=u, v$ and $T, T$ is the temperature, $\lambda=v$ if $f=u$ and $v, \lambda=k$ if $f=T, v$ is the viscous coefficient, $k$ is the thermal conductivity.

Using the definition (1) to simplify full Navier-stokes(NS) equations, we can deduce the equations governing ISF, that is for two-dimension compressible flow

$$
\frac{\partial}{\partial t}\left(\begin{array}{l}
\rho \\
\rho u \\
\rho v \\
\rho e_{t}
\end{array}\right)+\frac{\partial}{\partial x}\left(\begin{array}{l}
\rho u \\
\rho u^{2}+p \\
\rho u v \\
\left(\rho e_{t}+p\right) u
\end{array}\right)+\frac{\partial}{\partial y}\left(\begin{array}{l}
\rho v \\
\rho u v \\
\rho v^{2}+p \\
\left(\rho e_{t}+p\right) v
\end{array}\right)=\frac{\partial}{\partial y}\left(\begin{array}{l}
0 \\
\mu \frac{\partial u}{\partial y} \\
\frac{4}{3} \mu \frac{\partial u}{\partial y} \\
\mu u \frac{\partial u}{\partial y}+k \frac{\partial T}{\partial y}
\end{array}\right)
$$


Where $e_{t}=\frac{1}{\gamma-1} \frac{p}{\rho}+\frac{1}{2}\left(u^{2}+v^{2}\right), U$ and $V$ are velocity components in the streamwise, i.e. x-direction and normal direction of the dividing flow surface, respectively, $p$ and $\rho$ are the pressure and density, respectively. The equations(2) are just diffusion parabolized NS(DPNS) equations ${ }^{[6]}$, that are also called usually parabolized NS(PNS) equations or thin-later(TL) NS(TLNS) equations, that were presented by several authors directed against several concrete flows between the late 1960s and the late 1970s. The PNS equations are used widely in flow simulations for engineering purpose $\mathrm{e}^{[2,7]}$.

The ISF represents many typical flows, such as stagnation point flow, leading edge of a flat plate in a hypersonic flow, mixing layer flow with a strong transverse pressure gradient, flow along a streamwise corner, duct flow along the duct axis, viscous/inviscid interacting flow close neighbor wall surface etc. A further discussion is given in the next section.

\section{2 viscous/inviscid interacting shear perturbed flow(ISPF) theory ${ }^{[4,8]}$}

The ISPF as a result of perturbing ISF consists of viscous perturbed thin layer layer and its neighboring outer inviscid perturbed flow, describing which needs to use ISF's optimal coordinates. For unperturbed flow variables, the motion law and mathematical definition of the viscous perturbed layer are consistent with those of ISF's viscous layer. For perturbed flow, the motion-law and mathematical definition of viscous perturbed layer are similar to those of viscous unperturbed layer, then we 
have for two-dimensional incompressible flow

$$
\bar{u} \frac{\partial f}{\partial x} ? \quad \lambda \frac{\partial^{2} q}{\partial x^{2}}, \quad-\frac{\partial q}{\partial y} \cong \lambda \frac{\partial^{2} q}{\partial y^{2}}
$$

where $\uparrow=\tilde{u}, \tilde{v}$ or $\square, \tilde{u}$ and $\tilde{v}$ are perturbed velocity components, $T$ is the perturbed temperature, $\bar{u}$ and $\bar{v}$ are unperturbed velocity components, $\lambda$ 's definitions are given in the formula(1). Under the hypothesis of that both unperturbed variables $\overrightarrow{\vec{u}}$ and the sum of $\overrightarrow{\vec{u}}$ and perturbed variables $\overrightarrow{\tilde{u}}$ satisfy NS equations, we can deduce two sets of equations governing unperturbed and perturbed variables and use the formula(4) to simplify them and then we obtain ISPF equations governing unperturbed variables, that are consistent with those of the laminar ISF and other ISPF equations governing perturbed variables, that are

$$
\begin{gathered}
\left.\frac{\partial \vec{u}}{\partial t}+\stackrel{\vec{u}}{(\vec{u}} \cdot \nabla\right) \overrightarrow{\tilde{u}}+\underset{(\vec{u} \cdot \nabla)}{\vec{u}}+\frac{1}{\rho} \nabla p=\gamma \frac{\partial^{2} \overrightarrow{\tilde{u}}}{\partial y^{2}} \\
\operatorname{div} \overrightarrow{\vec{u}}=0
\end{gathered}
$$

The equations(5) were presented by Herbert ${ }^{[9]}$ in 1987 countering non parallel effects of the stability of boundary layer flow, that is only a case of various ISPF. Herbert called it parabolized stability equations(PSE). However, mathematical property of two-dimensional compressible PSE is consistent with that of DPNS equations, that is only parabolized diffusively. The name of PSE is incorrect, PSE ought to be substituted with diffusion parabolized stability equations(DPSE).

\section{3 viscous/inviscid interacting shear turbulent flow(ISTF) theory ${ }^{[4,10]}$}


The ISTF is similar to ISF. The ISTF consists of viscous turbulent layer and its neighboring outer inviscid turbulent flow. The viscous turbulent layer is convective-diffusion competitive in its normal direction and convection-dominate in its streamwise direction. If we describe incompressible turbulent flow by the Reynolds average NS(RANS) equations written in the ISF's optimal coordinates, then the motion-law and mathematical definition of ISTF's viscous turbulent layer are

$\bar{v} \frac{\partial \bar{f}}{\partial y} \cong \frac{\partial}{\partial y}\left(\lambda \frac{\partial \bar{f}}{\partial y}-\overline{v^{\prime} f^{\prime}}\right), \bar{u} \frac{\partial \bar{f}}{\partial x} ? \frac{\partial}{\partial x}\left(\lambda \frac{\partial \bar{f}}{\partial x}-\overline{u^{\prime} f^{\prime}}\right), \bar{w} \frac{\partial \bar{f}}{\partial z} ? \frac{\partial}{\partial z}\left(\lambda \frac{\partial \bar{f}}{\partial z}-\overline{w^{\prime} f^{\prime}}\right)$

where $\bar{f}=(\bar{u}, \bar{v}, \bar{w}, \bar{T}), \bar{u}, \bar{v}$ and $\bar{w}$ are time-average velocity component, $x, y$ and $z$ are the coordinates of the ISF's optimal coordinates, $\bar{T}$ is time-average temperature, $\lambda=v$ if $\bar{f}=\bar{u}, \bar{v}$ and $\bar{w}, \lambda=k$ if $\bar{f}=\bar{T}, v$ and $k$ are vicious coefficient and thermal conductivity, respectively.

Using the definitions(7) we can simplify RANS equations and then obtain simplified RANS equations governing ISTF

$$
\begin{gathered}
\frac{\partial}{\partial t}(\overrightarrow{\bar{u}})+(\overrightarrow{\bar{u}} \cdot \nabla) \overrightarrow{\bar{u}}+\frac{1}{\rho} \nabla \bar{p}=\frac{\partial}{\partial y}\left(v \frac{\partial \bar{u}}{\partial y}-\overline{v^{\prime} \overline{u^{\prime}}}\right) \\
\frac{\partial \bar{T}}{\partial t}+(\overrightarrow{\bar{u}} \cdot \nabla) \bar{T}+=\frac{\partial}{\partial y}\left(\frac{\lambda}{\rho c_{p}} \frac{\partial \bar{T}}{\partial y}-\overline{v^{\prime} T^{\prime}}\right) \\
\operatorname{div}(\overline{\bar{u}})=0
\end{gathered}
$$

where $\stackrel{\underline{v}}{u}=(\bar{u}, \bar{v}, \bar{w}), \quad c_{p}$ is the isobaric specific capacity. The ISTF equations(8) are the counterpart of ISF equations for laminar flow. The ISTF equations(8) contains unknowns $\overline{v^{\prime} T^{\prime}}$ etc. for which some 
additional equations or say turbulence models(such as the turbulence $k-\varepsilon$ models) must be supplemented. The $k-\varepsilon$ models corresponding ISTF equations are

$$
\begin{aligned}
& \stackrel{\vec{u}}{(\vec{u}} \nabla) k=\frac{\partial}{\partial y}\left[\left(v+\frac{v_{t}}{p_{v k}}\right) \frac{\partial k}{\partial y}\right]+\frac{\tau_{t}}{\rho} \frac{\partial \bar{u}}{\partial y}+S_{k}, \\
& (\overrightarrow{\vec{u}} \cdot \nabla) \varepsilon=\frac{\partial}{\partial y}\left[\left(v+\frac{v_{t}}{p_{v \varepsilon}}\right) \frac{\partial \varepsilon}{\partial y}\right]+\frac{\tau_{t}}{\rho} \frac{\partial \bar{u}}{\partial y}+S_{\varepsilon}
\end{aligned}
$$

where $k$ is the kinetic energy of the turbulent fluctuations, $k=\frac{1}{2}\left(\overline{u^{\prime 2}+v^{\prime 2}+w^{\prime 2}}\right), \quad \varepsilon$ is the dissipation, $v_{t}$ is the turbulent viscous coefficient; $\tau_{t}$ is the turbulent shear stress, $\tau_{t}=-\rho \overline{u^{\prime} v^{\prime}} ; p_{v k}$ and $p_{v \varepsilon}$ are the turbulent Prandtl numbers; $S_{k}$ and $S_{\varepsilon}$ are the turbulent source terms, that contain empirical constants, $C_{\varepsilon}$ is the empirical constants.

2. Inference I: Scaling-laws of velocity and length of ISF's viscous layer flow were given by Z.Gao ${ }^{[4,11]}$. The velocity and length scales of a three-dimensional incompressible flow can be expressed generally as

$$
\left(x_{p}, y_{p}, z_{p} ; u_{p}, v_{p}, w_{p}\right)=\left(R_{e}^{-n_{x}}, R_{e}^{-n_{y}}, R_{e}^{-n_{z}} ; R_{e}^{-n_{u}}, R_{e}^{-n_{v}}, R_{e}^{-n_{w}}\right)
$$

where $\left(x_{p}, y_{p}, z{ }_{p}\right)=(x, y, z) / L,\left(u_{p},{ }_{p}, w_{p}\right)=(u, v, w) / U, R_{e}=\rho u L / \mu$. Using the continuity equation and a hypothesis of that the variation of kinetic energy along the streamwise direction( i.e. the x-direction) in ISF's viscous layer, is independent of $R_{e}$, we can deduce 


$$
\left(n_{x}, n_{y}, n_{z} ; n_{u}, n_{v}, n_{w}\right)=\left(\frac{3}{2} q, \frac{(1+q)}{2}, n_{z} ; \frac{1}{2} q, \frac{(1-q)}{2}, n_{w}\right), n_{z}-n_{w}=q
$$

where the interacting parameter $q=\ln \frac{u_{p}}{x_{p}} / \ln R_{e}, 0 \leq q<\frac{1}{2}$; If $w$ can be compared with $u$, when $q=0$ ISF expresses the stagnation flow or the classical boundary-layer flow and its neighboring outer inviscid flow, between which there is no interaction; when $q=\frac{1}{4}$ the ISF's viscous layer is just the lower deck of the well-known Triple-deck theory ${ }^{[1]}$, in this case ISF express flow in neighborhood of separation point or reattachment point or tail-edge point or leading edge point or small step , hump, dents and chinks on wall surface etc. when $q=\frac{1}{2}$ the length scales and velocity scales of ISF's viscous layer are the same in all directions, i.e. an isotropic viscous flow. Therefore, the interacting parameter $q$ is essentially a measure of strength of viscous/inviscid interaction. In addition, if the effects of the Mach number or say compressibility are not neglected, we can deduce further the scaling-laws of the density and temperature, adding which to know scaling-laws.

ISF can express some typical flows mentioned above. The whole viscous/inviscid interacting flow in the neighborhood of wall surface is obviously a complex ISF, whose governing equations are DPNS equations written in a fitted body orthogonal coordinates, which is just a fitted dividing flow surface orthogonal coordinates or say the complex ISF's optimal coordinates. The scaling laws of velocity and length of 
ISPF's viscous perturbed flow are consistent with those of ISF's viscous flow $^{[8]}$.

The scaling-laws of velocity and length of ISTF's viscous turbulent flow are consistent with those of ISF's viscous layer flow if in the latter's scaling-laws $v$ is substituted with an effective viscous coefficient $v_{\text {eff }}$, where $v_{\text {eff }}=v+v_{t}, \quad v_{t} \frac{\partial \bar{u}}{\partial y}=-\overline{u^{\prime} v^{\prime}}$

\section{Inference II : Dividing flow surface criteria ${ }^{[12,13]}$}

In ISF's viscous layer there is always a dividing flow surface ${ }^{[1]}$, on which the normal velocity vanishes. The ISF equations, i.e. DPNS equations can be simplified further on the dividing flow surface and the resultant equations for a two-dimensional compressible flow are

$$
\begin{aligned}
& \rho u \frac{\partial u}{\partial x}+\frac{\partial p}{\partial x}-\frac{\partial}{\partial x}\left(\mu \frac{\partial u}{\partial y}\right)=0, \quad \frac{\partial p}{\partial x}-\frac{\partial}{\partial x}\left(\mu \frac{\partial v}{\partial y}\right)=0 \\
& \rho c_{p} u \frac{\partial T}{\partial x}-u \frac{\partial p}{\partial x}-\frac{\partial}{\partial y}\left(k \frac{\partial T}{\partial y}\right)-\mu\left(\frac{\partial u}{\partial y}\right)^{2}=0
\end{aligned}
$$

The resultant equations are called the dividing flow surface criteria, this is because that they can be used to verify creditability of numerical simulations for ISF. Two important special case of the dividing flow surface criteria are wall-surface criteria (WSC) for viscous flow and inviscid flow. This two WSC are respectively 


$$
\begin{array}{ll}
\left(\frac{\partial p}{\partial x}\right)_{w}-\frac{\partial}{\partial y}\left(\mu \frac{\partial u}{\partial y}\right)_{w}=0,\left(\frac{\partial p}{\partial y}\right)_{w}-\frac{\partial}{\partial y}\left(\mu \frac{\partial v}{\partial y}\right)_{w}=0 & (W M C) \\
\frac{\partial}{\partial y}\left(k \frac{\partial T}{\partial y}\right)_{w}+\mu\left(\frac{\partial u}{\partial y}\right)_{w}^{2}=0 & \text { (WEC) }
\end{array}
$$

and

$$
\begin{aligned}
& \left(\rho u \frac{\partial u}{\partial x}\right)_{w}+\left(\frac{\partial p}{\partial x}\right)_{w}, \quad\left(\frac{\partial p}{\partial y}\right)_{w}=0 \\
& \left(\rho c_{p} u \frac{\partial T}{\partial x}\right)_{w}-\left(u \frac{\partial p}{\partial x}\right)_{w}=0
\end{aligned}
$$

where WMC and WEC are wall momentum criteria and wall energy criteria, respectively. They can be used to verify creditability of numerical simulations for viscous flow or inviscid flow near wall surface.

The WSC for ISPF's unperturbed variables are consistent with those of ISF. The WSC for ISPF's perturbed variables are as follows ${ }^{[4,13]}$

$$
\begin{array}{ll}
\nabla p_{w}-\left[\gamma \frac{\partial^{2}}{\partial y^{2}}(\overrightarrow{\tilde{u}})\right]_{w}=0 & (W M C) \\
\frac{\partial}{\partial y}\left(\lambda \frac{\partial T}{\partial y}\right)_{w}+\mu\left[\left(\frac{\partial \tilde{u}}{\partial y}\right)^{2}+\left(\frac{\partial w}{\partial y}\right)^{2}\right]_{w}=0 & (W E C)
\end{array}
$$

The WSC(18) and (19) can be used to verify creditability of numerical results given by computing DPSE, i.e. PSE.

The WSC for ISTF's Reynolds time average variables are as follows $^{[4,13]}$ 
$(\nabla \bar{p})_{w}=\frac{\partial}{\partial y}\left(v \frac{\partial \vec{u}}{\partial y}-\overline{v^{r} u}\right)_{w}$

$\frac{\partial}{\partial y}\left(k \frac{\partial \bar{T}}{\partial y}\right)+\left[\mu\left(\frac{\partial \bar{u}}{\partial y}\right)^{2}+\left(\frac{\partial \bar{w}}{\partial y}\right)^{2}\right]_{w}-\rho c_{p} \frac{\partial}{\partial y}\left(\overline{v^{\prime} T}\right)_{w}+\frac{\partial}{\partial y}\left(\overline{v^{\prime} p^{\prime}}\right)_{w}=0 \quad$ (WEC)

The WSC for the turbulence models(such as the $k-\varepsilon$ turbulence models) are

$$
\begin{aligned}
& \frac{\partial}{\partial y}\left[\left(v+\frac{v_{t}}{p_{v k}}\right) \frac{\partial k}{\partial y}\right]_{w}+\left(\frac{\tau_{t}}{\rho} \frac{\partial \bar{u}}{\partial y}\right)_{w}+\left(S_{k}\right)_{w}=0 \\
& \frac{\partial}{\partial y}\left[\left(v+\frac{v_{t}}{p_{v \varepsilon}}\right) \frac{\partial \varepsilon}{\partial y}\right]_{w}+\left(c_{\varepsilon} \frac{\varepsilon}{x} \frac{\tau_{t}}{\rho} \frac{\partial \bar{u}}{\partial y}\right)_{w}+\left(S_{\varepsilon}\right)_{w}=0
\end{aligned}
$$

The WSC for the average variables can be used to verify creditability of RANS numerical solution for turbulence flow. The WSC for the turbulence models can be used to verify creditability of the turbulence models(equations) numerical solution and also to evaluate good and bad of different models and also to optimize the turbulence models and turbulent empirical constants from WSC's angles.

\section{Some applications of ISF theory and inferences to CFD}

\subsection{Application of ISF's optimal coordinates to grid design}

For computations of ISF and flow near wall surface, an optimal grid should be an orthogonal grid with grid-line paralleling with the coordinate axes of ISF's optimal coordinates. One example is to compute two-dimensional incompressible mixing layer flow, refer to Fig.1-4. Four grid systems are used, one is an optimal orthogonal grid the other's are the resultants of the optimal grid rotating anticlockwise $15^{\circ}, 30^{\circ}$ and 
$45^{\circ}$. The maximum gradients of velocity and temperature given by the latter are respectively about $40 \%$ and $50 \%$ of those given by the former grid. Thus errors influence the transit of momentum and energy across ISF's viscous layer and also affect the flow in downstream.

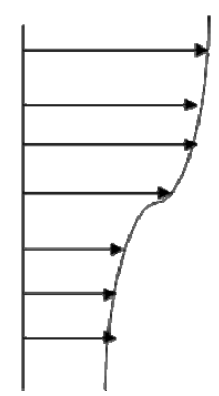

Fig.1 2-D mixing flow

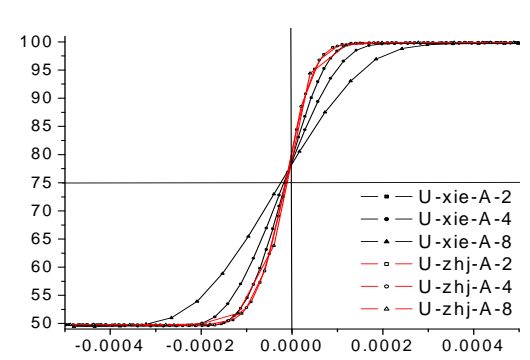

Fig.3 Velocity distributions

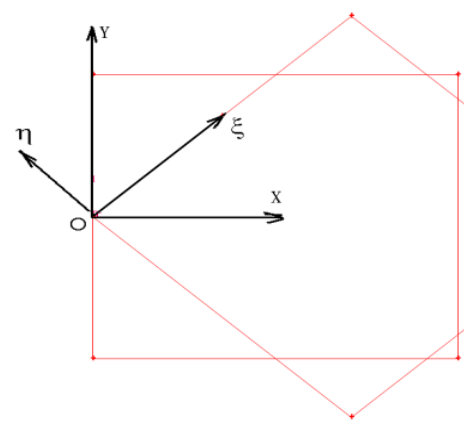

Fig.2 Optimal and non-optimal grid lines

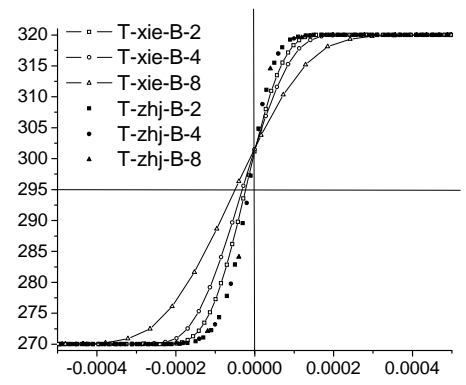

Fig.4 Temperature distributions

Therefore, in order to compute exactly various ISF occurring in flow

field computed, we ought to use ISF's optimal grid each individual, such as a base free ISF between base circulatory flow and outer inviscid flow and a complex free ISF between circulatory flow in separation region and outer inviscid flow and a complex viscous/inviscid interacting flow in the neighborhood of wall surface. Obviously, the three ISF's optimal orthogonal grids are completely different. For the complex ISF in the neighborhood of wall surface, the complex ISF's optimal coordinates are just fitted body orthogonal coordinates, having to use which in computing 
flow over a body is well-known to all.

\subsection{Application of the length scaling-laws of ISF's viscous layer to choose grid size}

The grid size is responsible for accuracy of numerical simulations. For the direct numerical simulation of turbulent flows, Kolmogorov scale is an important reference scale to choosing grid size. However, for an ordinary computation of NS equations, there was no definite method or standard to choosing grid size in the past. Obviously, the length scaling-laws given by ISF's theory would be a definite method to determine grid size, that have been proved tentatively by two sets of computation solving NS equations ${ }^{[14]}$.

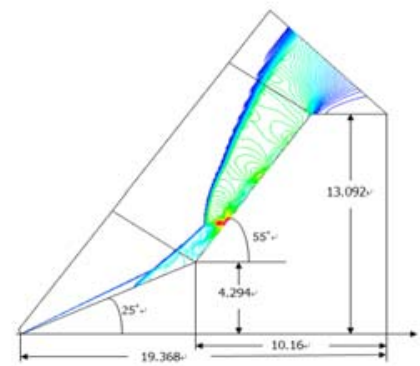

Fig. 5: SDC's computational domain and pressure contour

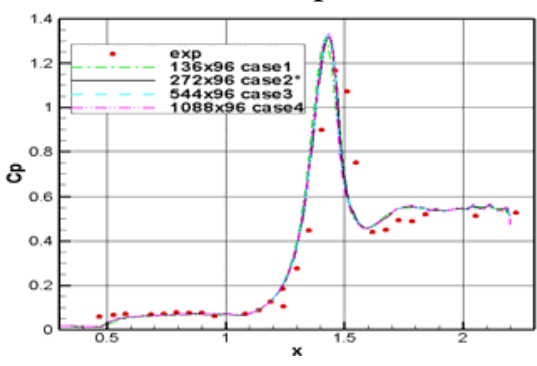

Fig. 7: Surface pressure coefficient for Run 8 over HCEF

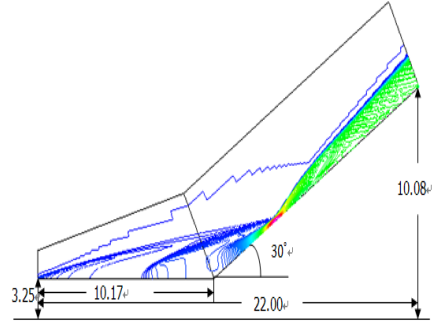

Fig. 6: HCEF's computational domain and pressure contour

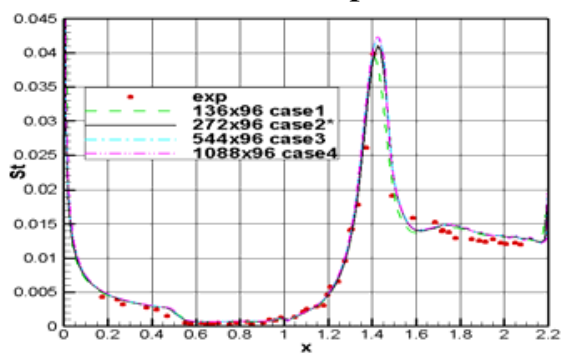

Fig. 8: Surface Stanton number for Run 8 over HCEF 


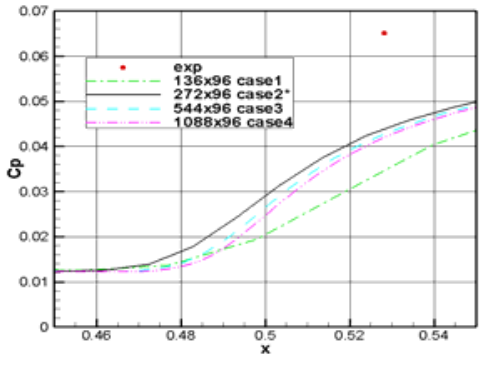

Fig. 9: Detail of Figure 3 around separation point

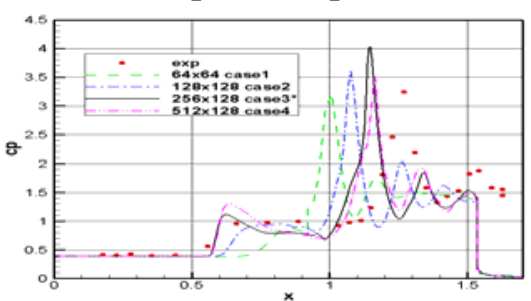

Fig. 11. Surface pressure coefficient for Run 28 over SDC

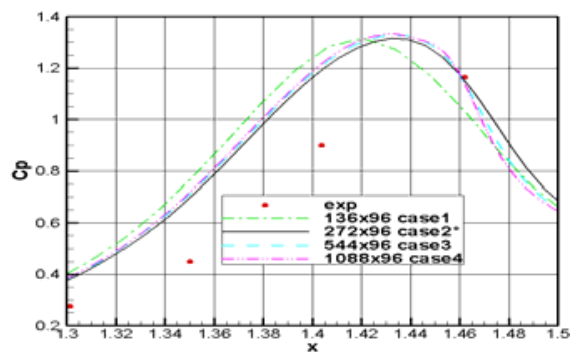

Fig. 10: Detail of Figure 3 near attachment point

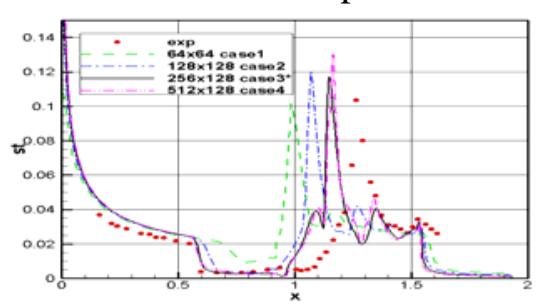

Fig. 12.Surface Stanton number for Run 28 over SDC

An analysis for the numerical results of hypersonic flows over both an asymptotic hollows cylinder extended flare(HCEF) and a sharp double cone(SDC) shows ${ }^{[14]}$ that in this two examples the better numerical solutions can be obtained(refer to Fig.5-12) when the grid sizes in both streamwise and normal directions are directly chosen as $1 / 10$ of the length scale with $q=1 / 4$ (see formula (10)and (11)), that can avoid refining repeatedly grid for seeking the best grid size. It should be emphasize on the importance of both ISF's optimal coordinates and the length scaling-law of ISF's viscous layer to grid design. Just as Schlichting and Gerstin pointed out in their monograph ${ }^{[12]}$ : "Numerical methods in computing flows at high Reynolds numbers only become efficient if the particular layered structure of the flow, as given by the asymptotic theory, is taken into account, as occurs if a suitable grid is used for computation.” 
Obviously, ISF theory shows further that numerical computations of high Reynolds number flow only become efficient if the thin-layered structure in all directions, given by ISF theory, is taken into account, as occur if a suitable grid with grid-lines paralleling the coordinate axes of ISF's optimal coordinates and with grid-refined locally according to the length scale of the small-scale structure in ISF's viscous layer is used for computations.

\subsection{Application of small-scale-structure to predict sudden changes of}

\section{heat flux etc.}

The scaling laws of ISF's viscous layer reveal that small-scalestructure generate certainly in ISF's viscous layer flow, that will do induce sudden changes of several physical quantities like wall heat flux and pressure gradient etc. as an example, for the case of laminar ISF and

Reynolds number $R_{e}=10^{6}$, the local wall heat flux is about 5.5 times if the interaction parameter $q=1 / 4$ and about 33 times if $q=1 / 2$ of wall heat flux given by the classical boundary-layer theory.

As far as I know that to date there no very convincing numerical results of using ISF's scaling laws to compute sudden increase of local heating rates etc. However, we ought to pay highly attention to thus computations for sudden change phenomena. This is because that some flight accidents, such as the damage of the rocket-powered X-15 when its flight velocity reached Mach 6.7 in 1967 and the demise of the space 
shuttle orbiter Columbia during its reentry from orbit in 2003. These accidents show that how severe the aerothermodynamic environment is for a vehicle that is traveling at hypersonic speeds and that how fragile the vehicles that fly through these environments can be. Analysis about mentioned above flight accidents making Bertin and Cummings ${ }^{[6]}$ giving the following conclusions: These locally severe, critical heating rates or unexpected deviations to the force and moments acting on the vehicle often occur due to viscous/inviscid interactions. These critical environments are the result of "unknown-unknown" or "gotchas". In fact, the scaling-laws of ISF's viscous layer have illustrated the mechanism of the sudden change phenomena of heating rates etc. and offered a way to compute locally sudden change.

\subsection{Application of the wall-surface criteria to verify creditability of NS numerical solutions}

The existence and uniqueness of the solution of NS equations have not been proved. So we have to face to an always confused problem--is it worth to trust the results from computer codes? If it does, how much can we trust it? Verification and validation of computing results become a very important work. The dividing flow surface criteria and wall surface criteria (WSC) are undoubtedly theoretical methods of verifying creditability of NS numerical solution. Z.Gao ${ }^{[11]}$ proved that eleven well-known NS exact solutions for incompressible flow satisfy exactly 
WSC and that both the solution of the classical boundary layer and its outer inviscid flow and the solutions of similar boundary layer with its outer inviscid flow satisfy WSC and that the local solution of ISF with $q=1 / 4$, i.e. the solution of Triple-deck theory ${ }^{[12]}$ satisfies WSC. Therefore, NS numerical solutions also ought to satisfy WSC, which are proved numerically by some computations of two-dimensional incompressible stagnation flow, shock/boundary layer interacting flow, compression ramp and cylinder flare ${ }^{[15-17]}$, refer to Fig,13-Fig.16.

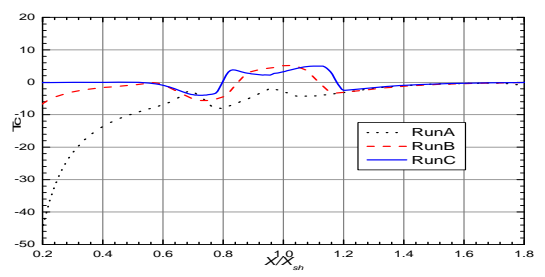

Fig.13 WMC in shock-boundary layer interacting flow

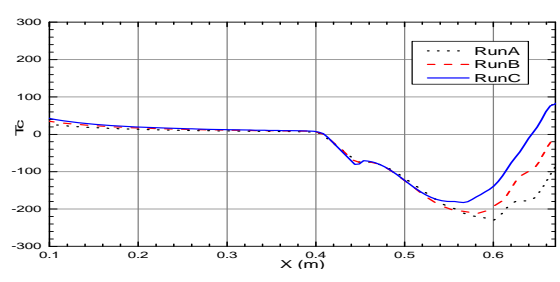

Fig.15 WMC in a viscous/inviscid interacting flow over ramp

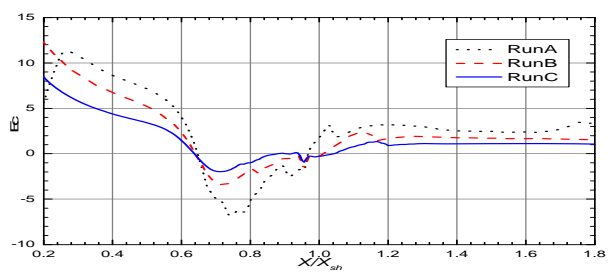

Fig.14 WEC in shock-boundary layer interacting flow

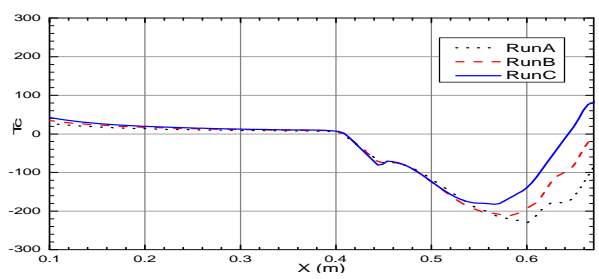

Fig.16 WEC in a viscous/inviscid interacting flow over ramp

In a word, we can obtain NS grid independent solution by operation of NS numerical calculations satisfying progressively the wall surface criteria(WSC). Especially, this criteria can evaluate the departure of NS numerical solutions from NS true solution by just one time NS calculation on set of grid, which is an outstanding advantage of the WSC 
method compared with the grid convergence analysis method in common use, based on that some people $e^{[5,15-17]}$ suggested that the WSC method may be used as an ingenious substitute for the grid convergence analysis method and that the WSC method and ISF theory would be called Gao's criteria and Gao’s ISF theory, respectively.

\subsection{Applications of WSC for turbulent flow to CFD}

The wall surface criteria(WSC) for turbulent flow can be used to verify creditability of RANS numerical solutions for near wall turbulent flow. The WSC for turbulent models can be used to evaluated good and bad of turbulent models and to optimize turbulent model and turbulent experience constants from WSC's angle, for example, an optimized Prandtl mixing length is given ${ }^{[13]}$

$$
l_{o p}=\left(\frac{v y}{5.75 \rho u_{\tau}}\right)^{1 / 2}, \quad l_{p}= \begin{cases}0.4 y & y \leq y_{c} \\ 4 \sigma & y>y\end{cases}
$$

where $y_{c}=0.15 \sigma \square 0.20 \sigma, \sigma$ is thickness of boundary layer, $l_{p}$ is Prandtl mixing length, $l_{o p}$ is optimized Prandtl mixing length, $u_{\tau}$ is the friction velocity.

\section{Conclusion}

The viscous/inviscid interacting shear flow(ISF) consists of viscous shear layer and its neighboring outer inviscid flow, which interact on each other. So ISF theory makes a breakthrough the classical boundary layer theory, that treat viscous thin shear layer without interaction with its outer 
inviscid flow. The equations governing ISF are diffusion parabolized NS equations, i.e., PNS equations. Anderson ${ }^{[2]}$ reviewed "The PNS method is in very widespread use; indeed, it forms the basis of an industry-standard computer program, which is used by virtually all major aerodynamics laboratories and companies.” The equations governing perturbed variables of ISPF are diffusion parabolized stability equations (DPSE), i.e., PSE. Herbert ${ }^{[18]}$ pointed out that PSE method forms the basis of an industry-standard flow stability analysis.

However, in early studies of PNS and PSE, it is not very clear what kind of basic flows are described by PNS and PSE, there are no basic fluid theories corresponding to PNS and PSE. The ISF and ISPF theories furnish complete answer to this question. Especially, ISF and ISPF theories and their inferences also have some important applications to CFD as mentioned in Sec.4. The known applications of ISF theory and its inferences to CFD are fruitful and creativeness. So study and computation in this aspect are worth notice, deepening and widening.

\section{References}

1. Schlichting H,Gersten K. Boundary-Layer Theory[M]. Springer-Verlag, Berlin 2000

2. Anderson JD Jr. Hypersonic and High-Temperature Gas Dynamics (2 ${ }^{\text {nd }}$ ed.) [M] AIAA Education Series 2006

3. Z.Gao. Viscous/inviscid interacting shear flow theory. Acta Mechanica Sinica, 1990,22(1):9-19(in Chinese)

4. Z.Gao. Interacting shear flow(ISF) theory,diffusion parabolized NS equations and wall-surface criteria and the applications. Chinese Mechanics Abstracts, 2007,21(3):13-22(in Chinese)

5. Yong Yu. Gao's interacting shear flow (ISF) theory and its inferences and their 
applications in CFD[J]. To be published in Jour.BIT 2013,22(1):10-20

6. Z.Gao Hierarchial structure of simplifying Navier-Stokes equations and mathematical property of simplified Navier-stokes equations. Acta Mechanica Sinica,1988,20(2): 107-116(in Chinese)

7. Rubin SG,Tannehill JC. Parabolized/reduced Navier-Stokes computational techniques[C]. Annu.Rev. Fluid Mech. 1992, 24:117-144

8. Z.Gao. Invariability of convection-diffusion interacting scale structure. Acta Mechanica Sinica,1992,24(6):661-670(in Chinese)

9. Herbert T, Bertoloti, FP. Stability analysis of non-parallel boundary layer bull. American Phys.soc.198732(8): 2097-2112

10. Z.Gao. Two-dimensional viscous/inviscid interacting shear turbulent flow(ISTF) theory. Science in China Series A, 1992 22(6):605-615

11. Z.Gao. Strong viscous layer flow theory with application to viscous flow computation. Acta Aerodynamica Sinica, 2001,19(4):420-426(in Chinese)

12. Z.Gao. The wall-surface criteria with application to evaluate creditability of CFD simulation. Acta Aerodynamica Sinica, 2008,26(3):378- 393(in Chinese)

13. Z.Gao. Can the wall-surface-criteria method for substitute the grid convergence analysis method in common use? In:Proc.of 2012 Summer Symposium, State Key Laboratory of High Temperature Gas Dynamics(pp. 113-122)2012.8 yanji Jilin 2012(in Chinese)

14. Y.Yu, H.R.Zhang. A guidance to grid size design for CFD numerical simulation of hypersonic flows. Submitted to the $7^{\text {th }}$ Asian-Pacific Conference on Aerospace Technology and Science. May.2013 Taiwan

15. Y.Yu. New CFD validation method with application to verify computation of near wall flow[J]. Jour. Beijing Institute of Technology, 2010,19(3):259-263

16. X.W.Xu Wall-surface criteria-a method of evaluating creditability of CFD, analysis and application. Academic degree thesis, Beijing Institute of Technology, 2012(in Chinese)

17. Yong Yu. Gao's interacting shear flow (ISF) theory and its inferences and their applications in CFD[J]. To be published in Jour.BIT 2013,22(2)

18. Herbert T. Parabolized stability equations(PSE). Annu.Rev. Fluid Mech.1997,29:245-283 